\title{
La investigación científica aplicada a la caracterización de materiales y la selección de tratamientos de conservación
}

\author{
Jesús Espinosa Gaitán \\ Rosario Villegas Sánchez \\ Departamento de Análisis \\ Centro de Intervención IAPH
}

\section{Resumen}

Como apoyo al Proyecto de Investigación e Intervención para la restauración de la Puerta de Córdoba de Carmona, se ha realizado un estudio de laboratorio con dos objetivos:

- caracterización de materiales y de su estado de conservación, de cara a la investigación históricoarqueológica y a la definición del proyecto arquitectónico.

- evaluación del comportamiento de distintos tratamientos para piedra, con objeto de seleccionar los más adecuados para la intervención sobre el edificio.

\section{Palabras clave:}

Carmona / Puerta de Córdoba / Materiales de Construcción / Estudio Petrográfico / Consolidante / Hidrófugo / Ensayos Alteración

\section{Introducción}

Dentro del proyecto de conservación-restauración de la Puerta de Córdoba de Carmona se han realizado una serie de estudios y ensayos de laboratorio con dos objetivos: caracterizar los materiales y su estado de conservación, así como seleccionar los tratamientos más adecuados.

Por lo que se refiere a la primera parte, el estudio analítico se ha centrado fundamentalmente en la caracterización de los materiales tanto de las estructuras emergentes como de aquellos encontrados en

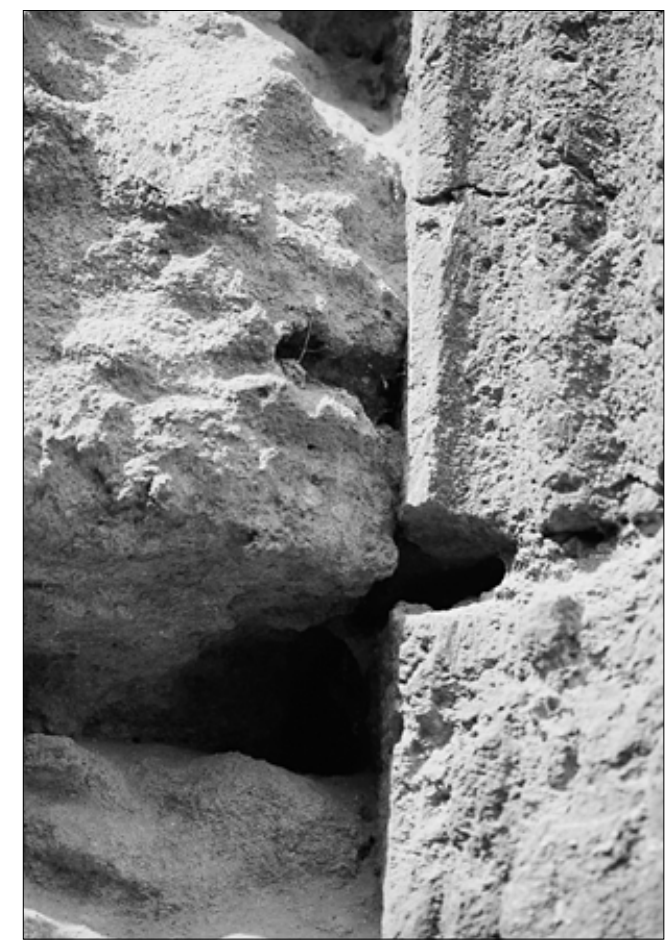

las excavaciones arqueológicas realizadas. El estudio se planteó con una doble finalidad; por un lado determinar los factores y causas de alteración presentes, con vistas a establecer los parámetros a controlar en la actuación, y por otro, obtener información de interés arqueológico.

La Puerta de Córdoba se localiza en el extremo NE del casco antiguo de la ciudad de Carmona, aprovechando una vaguada en el Alcor, siendo en su origen de época romana. Está construida fundamentalmente a base de sillares de piedra calcarenítica, los cuales en algunas zonas se encuentran revestidos con enfoscados y enlucidos pertenecientes a distintas intervenciones realizadas a lo largo de su historia. Además aparecen asociados con el conjunto de la estructura diversos lienzos de muralla. 
El material pétreo utilizado procede de la formación geológica de Los Alcores y corresponde a las típicas calcarenitas amarillentas de gran proliferación en las construcciones históricas de la zona.

En el momento de abordar el estudio, la piedra de la Puerta mostraba un avanzado estado de deterioro, con notables pérdidas de material por fenómenos de alveolización, arenización, fisuración y desplacados. Además, en numerosos sectores se apreciaba pérdida total o parcial de los revestimientos aplicados en épocas pasadas.

Los análisis se han efectuado sobre muestras de piedra de la Puerta, piedra de las posibles canteras de abastecimiento, morteros, murallas y materiales de revestimientos. Además, se tomaron bloques de piedra desprendidos de las murallas, con los que se prepararon probetas de ensayo para determinar algunas características físicas y se emplearon en la evaluación de tratamientos.

El estudio previo de los tratamientos que se utilizan en la restauración de bienes inmuebles debe realizarse para seleccionar aquéllos más adecuados a todos los factores que inciden sobre el edificio -condiciones climáticas, contaminación ambiental, uso, etc.- así como para los materiales pétreos que lo constituyen.

Los productos de tratamientos de piedra se dividen en dos tipos cuya aplicación tiene objetivos completamente distintos: consolidantes e hidrófugos (también llamados protectores). La aplicación de un producto consolidante es necesaria únicamente cuando la piedra ha perdido cohesión y debe introducirse un material que consiga la unión entre los granos minerales que la forman y que han quedado sueltos. Debe aplicarse de forma que consiga unir la zona alterada de la piedra, más o menos superficial, a la zona interna sana, por lo que una de las exigencias más importantes que deben cumplir es la de una buena penetración.

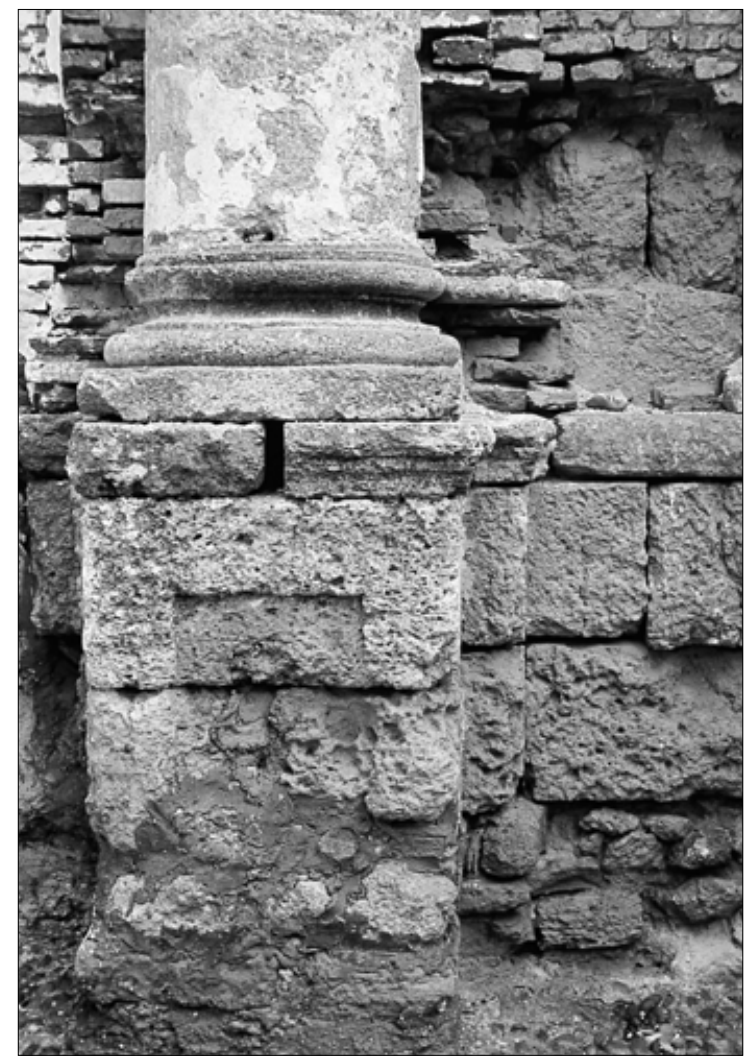

Fig. I Aspecto del alto grado de alteración de la piedra y reparcheos de intervenciones recientes
La hidrofugación, también llamada protección, se basa en la aplicación de un tratamiento que impida la entrada de agua líquida en la piedra pero que permita la salida del agua en forma de vapor, es decir, que mantenga la "respiración" del material.

En este caso se consideró necesario aplicar un tratamiento consolidante en todas aquellas zonas donde la piedra estuviese decohesionada y, posteriormente, un hidrófugo en las superficies donde fuese necesario proteger al material de la entrada de agua de lluvia. Para seleccionar los más adecuados se estudiaron diferentes características de la piedra tratada, siguiendo la metodología que se describe posteriormente. 


\section{CARACTERIZACIÓN DE LOS MATERIALES}

\section{Técnicas empleadas}

Las técnicas y métodos empleados para el análisis de las muestras en el presente estudio son las siguientes:

- Difracción de Rayos X (DRX) para la determinación mineralógica.

- Estudio de láminas delgadas mediante Microscopía óptica de Polarización.

- Análisis Químicos: Volumetría Gravimétrica, Espectrofotometría de Absorción Atómica y de Ultravioleta Visible.

- Estudio estratigráfico por microscopía óptica y microscopía electrónica con microanálisis por energía dispersada de Rayos X.

- Saturación de agua al vacío (porosidad abierta).

\section{Materiales pétreos}

\section{Contexto Geológico}

La ciudad de Carmona está ubicada en la denominada Depresión del Guadalquivir en el borde de la cornisa del Alcor, dentro del marco geológico de las que se han considerado Unidades Autóctonas regionales, concretamente sobre materiales del Mioceno Superior-Plioceno. Dentro de esta unidad se han distinguido de muro a techo tres formaciones geológicas:

- Margas azules y grises. Éstas afloran bordeando las zonas más deprimidas de la Cornisa del Alcor, siendo abundantes en las inmediaciones de Carmona. Son ricas en microfauna de foraminíferos planctónicos y poseen gran cantidad de elementos ferruginosos, de ahí su coloración amarillenta en superficie al alterarse. La potencia de esta formación puede llegar a ser superior a los 300 metros.

- Margas Azules y grises con Calcarenitas. Aparecen en aparente concordancia con las formaciones superior e inferior. Los afloramientos de esta forma-

Fig. 2 Cantera de la Batida.

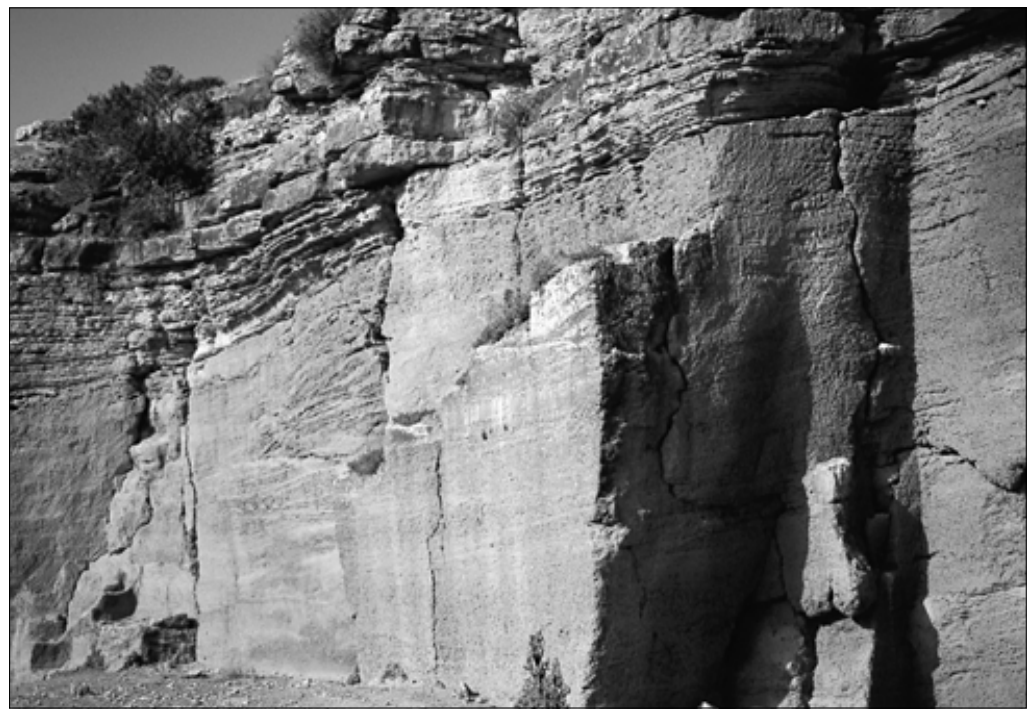

ción no son importantes debido a su escasa potencia $(10-15 \mathrm{~m})$, y a los frecuentes deslizamientos ocasionados por las calcarenitas superiores sobre las margas azules, a causa de la plasticidad de éstas cuando están húmedas. Sin embargo, sí existe buena representación de ellas en la inmediaciones orientales de Carmona. Al igual que en la anterior formación, también aparecen contenidos importantes de foraminíferos planctónicos.

- Calcarenitas, Areniscas y Limos amarillos. Los afloramientos de esta formación están muy bien representados, asentándose Carmona sobre ella en su mayor parte. Estos materiales aparecen de forma concordante con las anteriores y el paso entre ambas es gradual.

De forma habitual las calcarenitas presentan un aspecto masivo en el cual apenas se reconoce la estratificación. Por lo general son muy groseras (arena gruesa) y están poco a nada clasificadas. Se constituyen por al menos un $80 \%$ de bioclastos, y el resto por elementos siliciclásticos y minerales férricos que confieren el color amarillento al alterarse. A veces las calcarenitas aparecen con mejor estratificación cuando el sedimento es más fino (arena media), apareciendo estratos de aproximadamente $5 \mathrm{~cm}$ de espesor, entre los que puede haber láminas de lodo o cantos blandos arcillosos. Estos materiales presentan en general una microfauna muy pobre en foraminíferos planctónicos.

\section{Estudio Petrográfico}

Los materiales pétreos estudiados corresponden a varias muestras de la Puerta extraídas de distintos puntos y en diferentes estados de alteración, y muestras de cantera para establecer la posible procedencia de la piedra puesta en obra, así como comparar el estado de alteración en el edificio respecto a éstas.

Existe una gran cantidad de canteras de calcarenitas a lo largo de toda la formación de los Alcores (algunas abandonadas y otras en activo) debido a que esta zona ha sido un enclave muy favorable para el asentamiento humano a lo largo de la historia.

Las canteras estudiadas se han elegido siguiendo criterios arqueológicos y han sido las siguientes: cantera de la Necrópolis, pequeñas canteras romanas en desuso en los alrededores de la ciudad en la zona NE, cantera de La Batida (explotaciones modernas, pero es posible que se utilizaran en época romana), cantera del Castillo de Mairena del Alcor, y cantera del Puerto del Judío, que es la más importante explotación romana localizada en esta zona.

La composición mineralógica de las muestras estudiadas, ya sean procedentes del edificio o de cantera, es muy similar, apareciendo como componente mayoritario la calcita, con contenidos que oscilan entre el 60-85\%, acompañada de cuarzo con contenidos entre el 15-30\%. La variabilidad en el contenido de 
estos dos minerales es independiente de su procedencia, ya que dentro del edificio o de una misma cantera se pueden dar los márgenes extremos. Esto se debe a la presencia de niveles más detríticos cuanto mayor es el contenido en cuarzo, siendo muy elevado en todas las muestras de la cantera de la Batida (30-60\%).

En cantidades minoritarias $(<5 \%)$ se ha detectado en todas las muestras la presencia de dolomita, pero lo que resulta más significativo es también la presencia de yeso en todas ellas, siendo minoritario (<5\%) en muestras de cantera, pero llegando a ser importante en la mayoría de muestras del edificio (5-15\%).

Aunque con el método de polvo de DRX no se ha detectado la presencia de minerales de la arcilla dada la escasa proporción en que se encuentran, el aspecto de la piedra en algunas zonas y la presencia de nódulos manifestaba su presencia. Mediante agregados orientados se estudió la fracción fina y se ha constatado la existencia de minerales de la arcilla, mayoritariamente esmectitas e ilita.

Las esmectitas son arcillas expansivas que pueden llegar a duplicar su espaciado interlaminar fácilmente en presencia de humedad, hecho que podría explicar en parte la alteración tan acusada que en algunos casos se observa, a causa de las contracciones y dilataciones en las estructuras de las esmectitas.

Los análisis químicos realizados sobre la piedra muestran resultados en correspondencia con los obtenidos con DRX. En la tabla I se recoge la composición química de dos muestras de la Puerta.

De los datos obtenidos cabe resaltar el alto contenido en sulfatos en la muestra PCC3, que posiblemente corresponda a yeso, tal y como ha quedado confirmado mediante otras técnicas.

El estudio de láminas delgadas mediante Microscopia óptica pone de manifiesto que todos los materiales corresponden a un mismo litotipo, aunque pueden existir pequeñas diferencias mineralógicas y texturales. A grandes rasgos la roca está constituida por un entramado de bioclastos (70-90\%), esencialmente lamelibranquios de concha fina (que suponen el 90$95 \%$ de los bioclastos) y en escasa abundancia briozoos y foraminíferos, estando en la mayoría de los casos removilizados y fragmentados.

Estos bioclastos se encuentran parcialmente rodeados de una matriz fina carbonatada (micrita), existiendo también óxidos de Fe y ocasionalmente material detrítico fino (arcilloso). Además existe un cemento esparítico que rellena parcialmente las oquedades que quedan entre todo el entramado clástico.

En la mayoría de las muestras se observan evidencias de disolución tanto de la matriz y cemento carbonatados como de los restos de los bioclastos. Este material disuelto ha reprecipitado en los espacios vacíos de la roca con cristales de tamaño esparítico en forma de "mosaico", o en los casos más desfavorables, como en algunas muestras de la Puerta, ha podido migrar hacia el exterior quedando algunos sectores de la roca constituidos prácticamente por el entramado de clastos, viéndose de este modo la porosidad incrementada y con una notable pérdida de cohesión.

Además de los componentes carbonatados existe, con una abundancia variable (10-30\%), una fracción detrítica constituida fundamentalmente por granos de cuarzo de tamaño arena media-fina y formas redondeadas. Ocasionalmente también aparecen granos de feldespatos.

Todas las muestras estudiadas presentan yeso rellenando algunos poros, siendo su abundancia variable de unas a otras. Aunque el contenido en yeso es mayor en muestras procedentes de la Puerta, en algunas de la cantera del Alcor su contenido es considerable. La presencia de yeso en cantera indica la naturaleza intrínseca del mismo, pero las concentraciones tan elevadas encontradas el algunas muestras de la Puerta (hasta un 15\%), junto a un hábito de cristalización distinto, más fibroso, hace pensar, que al menos en parte, el yeso proceda de morteros de unión, o bien por movilización (disolución y reprecitación) del yeso de las zonas internas de la piedra a las superficiales.

\section{Tabla 1. Composición química de muestras de piedra de la Puerta}

\begin{tabular}{lccccccc}
\hline Muestra & Pérdida & $\mathrm{SiO}_{2}$ & $\mathrm{CaO}$ & $\mathrm{MgO}$ & $\mathrm{Fe}_{2} \mathrm{O}_{3}$ & $\mathrm{Al}_{2} \mathrm{O}_{3}$ & $\mathrm{SO}_{3}$ \\
\hline PCC2 & 38.10 & 9.09 & 48.87 & 0.32 & 0.98 & 0.26 & 3.23 \\
\hline PCC3 & 31.49 & 9.07 & 44.37 & 0.55 & 0.74 & 0.31 & 12.52 \\
\hline
\end{tabular}

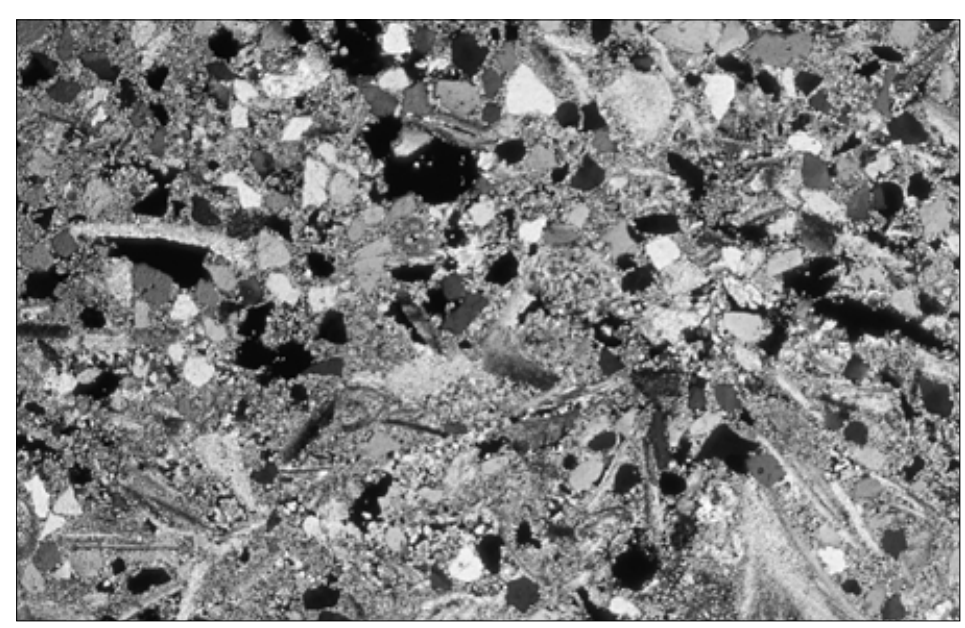

Fig. 3 Microfotografía de calcarenita del edificio en zona rica en

La porosidad de la roca observable mediante esta téccuarzo. nica (macroporosidad) es muy elevada, variando de una muestras a otras. Los poros aparecen bien intercomunicados y fundamentalmente son de tipo intergranular y móldicos. Mediante el ensayo de Saturación de agua al vacío (Van Keulen, 1972) se ha determina- 

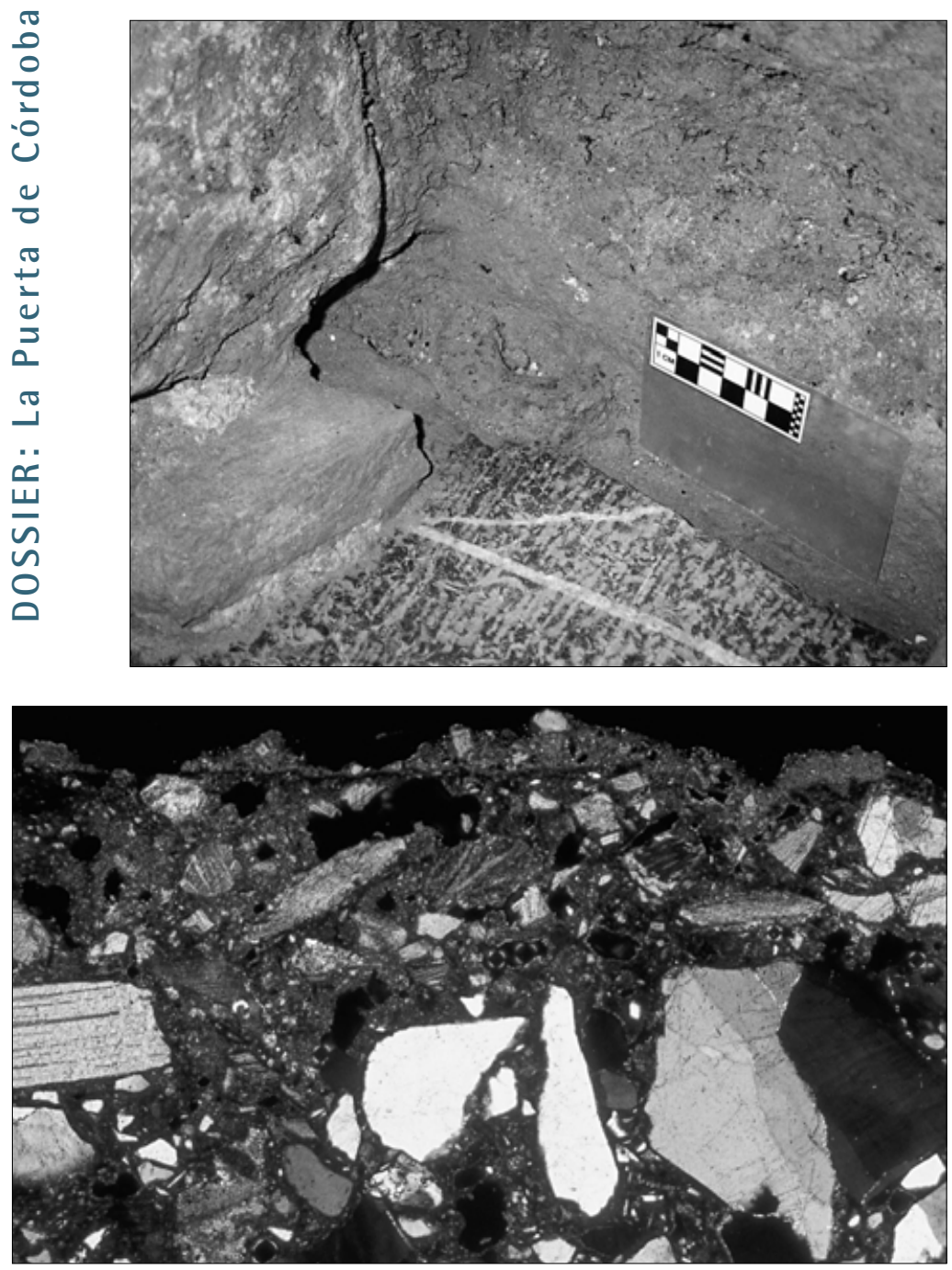

Fig. 4 Losa de piedra (caliza esparítica) en el Pasaje Norte.

Fig. 5 Microfotografía del anterior dónde se observan las dos capas.

Fig. 6 Revoco y enlucido romano conservado en la basa de una pilastra.

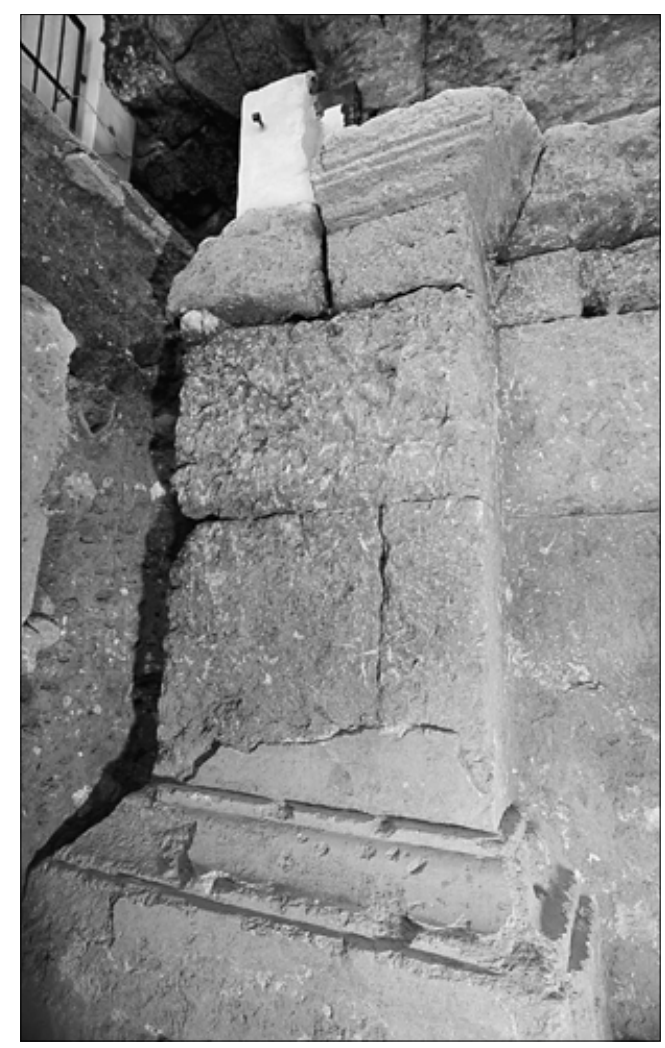

do la porosidad sobre 40 probetas obteniéndose valores que oscilan entre el $28 \%$ y el $40 \%$, estando la porosidad media en torno al 34\%, lo que pone de manifiesto que se trata de un material muy poroso. Por otro lado, y relacionado con las excavaciones arqueológicas, se han estudiado dos losas de piedra encontradas, una de ellas del Pasaje Norte (de época romana) y otra descontextualizada, con la intención de comprobar de si se trata del mismo material y poder atribuir su colocación al mismo periodo. Tras el estudio petrográfico se ha comprobado que aunque ambas son rocas cristalinas y compactas, pertenecen a litotipos distintos, la primera corresponde a una caliza esparítica de coloración oscura, y la segunda corresponde a un mármol blanco de grano fino.

\section{Morteros y revestimientos}

De este tipo de materiales se ha estudiado una gran diversidad de muestras considerando su función constructiva y las épocas de su puesta en obra según referencias arqueológicas.

- Morteros de unión de sillares de piedra. Sólo se han estudiado muestras adscritas al siglo XVIII, y corresponden con las únicas en las que se han detectado yeso en cantidades importantes en su composición, con porcentajes entre el 15-20\%. Además como componente mayoritario aparece calcita (65-75\%) y en menor cantidad cuarzo como árido (8-15\%). Se podrían considerar como morteros bastardos de cal y yeso, que podrían estar aportando sulfatos a la piedra.

- Morteros de reparación o recrecimiento de sillares. También de la misma época que los anteriores, son morteros de cal con composiciones muy sencillas a base de calcita (80-85\%) y cuarzo ( 15 $20 \%$ ), y que es muy probable que se elaboraran con materiales locales a base de albero (limos y arenas amarillentas) con adición de cal.

- Enfoscados y enlucidos romanos. Se han estudiado muestras de diferentes zonas de la Puerta y de las estructuras encontradas en las excavaciones arqueológicas. Algunos de estos materiales se estudiaron con el propósito de compararlos con las muestras de las que se tenía mayor certeza de su cronología. Tras los análisis, y dadas las peculiaridades petrográficas, no encontradas en otras muestras estudiadas, se han podido incluir como pertenecientes al mismo periodo.

Estos materiales se caracterizan por presentar claramente diferenciadas dos capas, una capa interna de gran espesor, perteneciente a un enfoscado de granulometría grosera, y un enlucido superficial de grosor más fino ( $1-3 \mathrm{~mm}$ ) y mejor acabado. En algunos casos el enlucido ha desaparecido o no se aplicaría en su momento.

El enfoscado interno presenta bajo contenido en cal (15-35\%) y un árido muy abundante (65-85\%) prácticamente constituido en su totalidad por granos de cuarzo de tamaño arena gruesa ( I-2 mm) y de formas muy angulosas. El estado de conserva- 
ción de esta capa es variable de unas muestras a otras, observándose grandes pérdidas de cal en algunas de ellas.

La composición mineralógica del enlucido exterior es prácticamente calcárea en su totalidad. Este nivel se compone de cal fina como aglomerante (aproximadamente el 40-50\%), y lo que resulta más significativo, presenta un árido que se compone de fragmentos de mármol de machaqueo (marmolina) con formas muy angulosas y tamaño de arena fina-media $(0,2-1 \mathrm{~mm})$. De forma minoritaria se observan pequeños granos de cuarzo, también anguloso, de tamaño muy fino $(0,1 \mathrm{~mm})$. Este enlucido es mucho más compacto que el enfoscado subyacente.

- Enfoscado adscrito como romano. Presenta características distintas a las anteriores, siendo petrográficamente de mayor similitud a los estudiados del Siglo XVIII. En ésta también se han diferenciado dos capas, una externa de muy poco grosor $(\mathrm{l} \mathrm{mm})$, y otra interna de mayor grosor.

La capa externa corresponde a un enlucido muy compacto, compuesto casi únicamente de cal, con árido escaso $(<10 \%)$ de cuarzo. La capa interna posee un aglomerante calcítico y áridos de distinta naturaleza, fundamentalmente cuarzo con formas subredondeadas y tamaño arena fina-media, y lo que es más destacable gran abundancia de fragmentos de roca calcarenítica similar a la de la Puerta.

Esto indica que para la elaboración de la parte interna de este enfoscado probablemente se emplearan arenas locales, mientras que en los anteriores la presencia de cuarzo anguloso y de marmolina apunta a una mayor selección en los áridos.

- Enfoscados atribuidos al Siglo XVIII. Presentan rasgos composicionales y texturales muy similares al último descrito, con proporciones de calcita entre el $50-70 \%$ y de cuarzo entre el $30-50 \%$.

Al igual que en el caso anterior se diferencian dos capas, una externa rica en cal y escaso árido de cuarzo, y otra interna más grosera con mayor proporción de árido, compuesto de cuarzo y fragmentos de calcarenitas con lamelibranquios.

- Aparte de las anteriores, se han estudiado varias muestras de enlucidos sin clara atribución cronológica, que por su composición y textura es difícil asignar a épocas concretas, ya que se componen en todos los casos prácticamente en su totalidad de cal (90\%) y árido de cuarzo muy fino (10\%)

- Revestimientos de color. Se han preparado estratigrafías de muestras de revestimientos coloreados que aparecían en el Pasaje Sur (de época romana) para determinar la superposición de estratos y la composición de los pigmentos utilizados en cada uno de ellos. La estructura y composición detectada es muy semejante en todas ellas (tabla 2).

\section{Tabla 2. Estratigrafías Revestimientos de color}

\begin{tabular}{ll}
\hline Capa I & $\begin{array}{l}\text { Capa de preparación blanca, a base de } \\
\text { carbonato cálcico }\end{array}$ \\
\hline Capa 2 & $\begin{array}{l}\text { Rojiza, a base de carbonato cálcico y } \\
\text { tierra roja (óxido de hierro) }\end{array}$ \\
\hline Capa 3 & Blanca, a base de carbonato cálcico \\
\hline
\end{tabular}

\section{Hormigones de las murallas}

1. Asociados al conjunto de la estructura de la Puerta de Córdoba aparecen varios lienzos de muralla, que en su mayor parte, según referencias arqueológicas, fueron levantados en el Bajo Medievo (siglos $\mathrm{XIV}-\mathrm{XV}$ ) y que se constituyen de hormigones de cal elaborados mediante la técnica del tapial.

Visualmente se han podido establecer diferencias entre tramos de muralla o entre diferentes tongadas de un mismo tramo, referidas a la fracción más grosera (gravas). Esta fracción más gruesa es muy variable en cuanto a tamaño y naturaleza, observándose en algunos casos cantos de piedra de hasta $40 \mathrm{~cm}$ de diámetro; en otros casos aparecen tongadas en las que los cantos no exceden los 7-8 cm. La naturaleza de estos cantos es variada, predominando respecto a los demás los de calcarenita local. Además de piedra,

Fig. 7 Aspecto de la muralla (tapial) en el adarve norte.

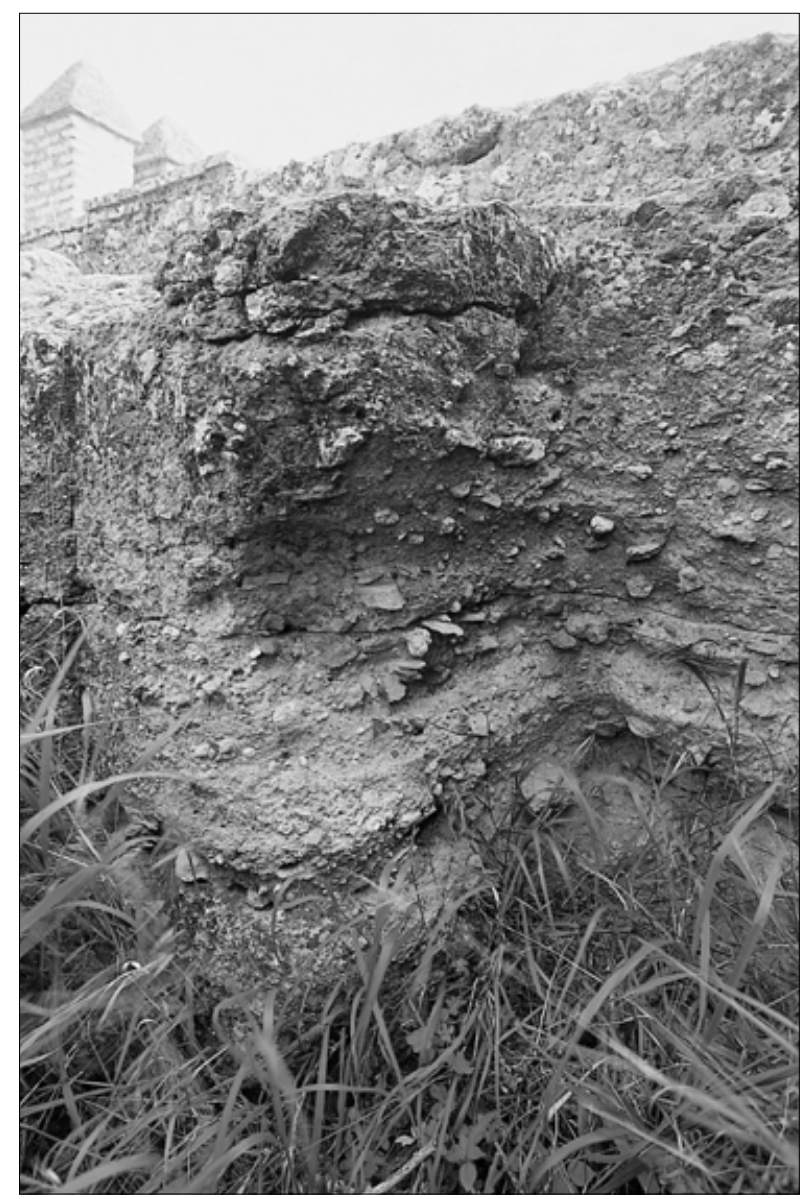




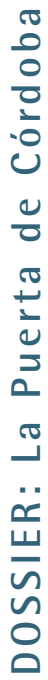

Fig. 8 Microfotografía de la matriz del tapial (adarve norte) con zonas de cal diferenciadas.

Fig. 9 Microfotografía de la matriz del núcleo del tramo norte de muralla.
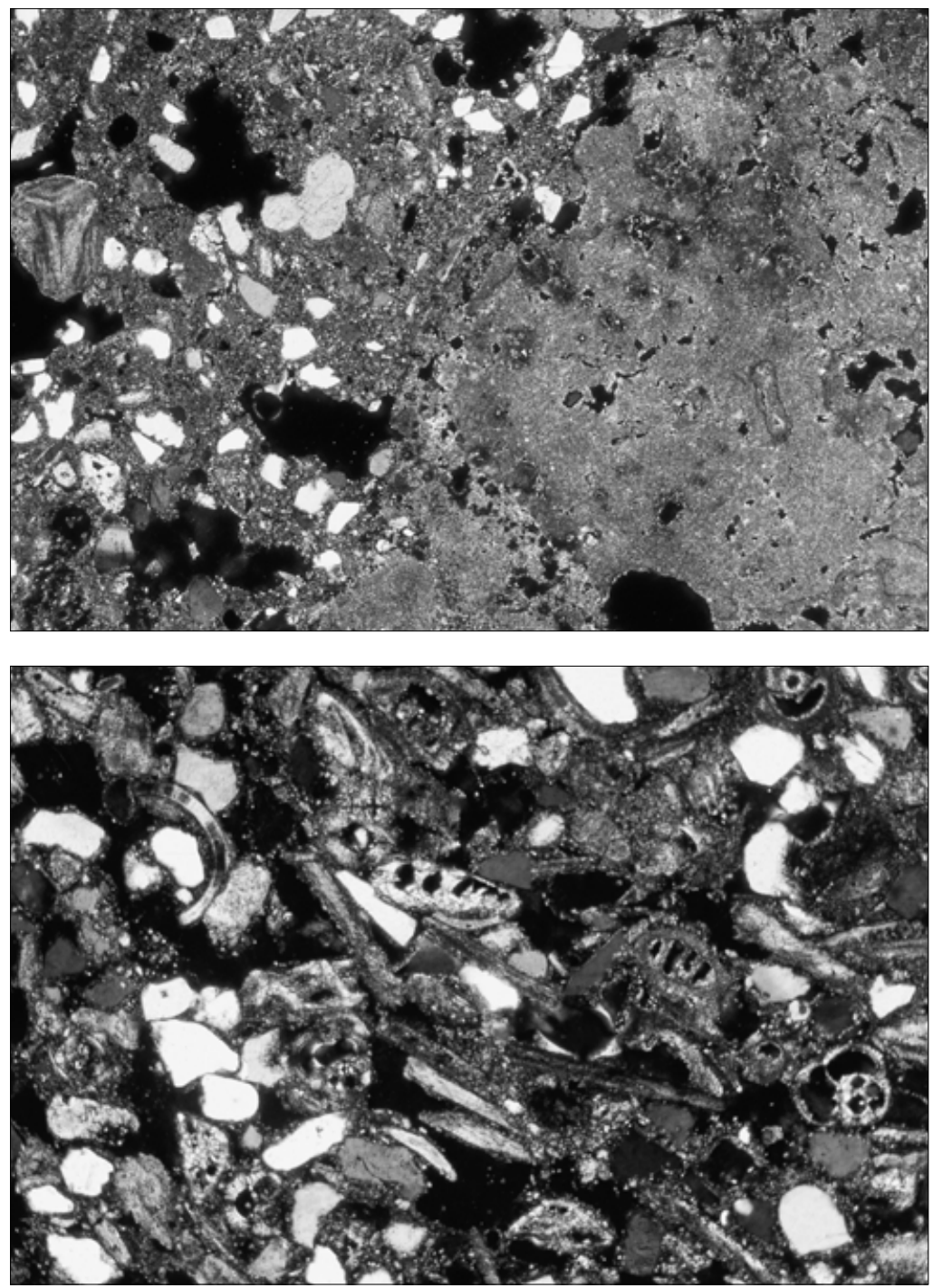

en determinados sectores se han identificado cascajos y fragmentos cerámicos de ladrillos.

Dada la complejidad de un estudio en detalle de la fracción más grosera de los tapiales, aparte de las evidentes diferencias macroscópicas, se pensó que se obtendría información más valiosa del estudio de la fracción más fina, en lo que se refiere a diferencias o analogías relacionadas con la naturaleza y proporción del aglomerante, y conocimiento de su textura. Así pues, de este tipo de materiales sólo se ha analizado la fracción más fina del tapial, que incluye, además del aglomerante, los áridos de granulometría más fina (tamaño arena), y que en su globalidad constituye lo que se podría considerar la matriz del tapial.

Se extrajeron muestras de la matriz de distintos tramos de tapial y/o de tongadas distintas con grandes diferencias granulométricas. La mineralogía de todas las muestras es muy similar, compuesta a base de calcita (60-70\%), cuarzo (30-40\%), feldespatos y filosilicatos en cantidades minoritarias.

Mediante el estudio de láminas delgadas se ha observado que las características composicionales y textude las piedras del edificio y de cantera estudiados, al que se le adicionó cal para conferir consistencia.

Así pues, parece ser que las principales diferencias entre los distintos tramos de muralla o entre tongadas radican en la naturaleza y granulometría de los cantos groseros.

2. Por otro lado también se han estudiado muestras del tramo Norte de la muralla, al parecer de época romana. Este tramo se caracteriza por la presencia alternativa de tongadas de cascajos, albero y limos amarillentos en distintas proporciones.

Se ha estudiado el nivel de limos del núcleo de la muralla, el cual pertenece también a un sedimento limoarenoso local, pero distinto de los anteriores dado su mayor contenido en arcillas, color más anaranjado por un mayor contenido en minerales férricos, y la presencia en gran abundancia de microfósiles (foraminíferos: globigerinas, miliólidos), que son muy poco frecuentes en las calcarenitas estudiadas y en las muestras de tapial anteriores.

Además, no se ha observado de forma clara la adición de cal a la hora de ponerlos en obra, y si la hubiera sus proporciones son mucho menores que en las muestras anteriores.

\section{EVALUACIÓN DE TRATAMIENTOS DE CONSERVACIÓN}

\section{Metodología de evaluación de tratamientos}

Para evaluar los tratamientos y seleccionar el (los) más adecuado(s) se ha seguido la metodología puesta a punto en el I.A.P.H. (Villegas, 1996), consistente en estudiar tres aspectos: 


\begin{tabular}{|c|c|c|c|c|}
\hline Producto & Fabricante & Composición & Efecto & Aplicación \\
\hline Tegovakon & Goldschmidt & Silicato de etilo & Consolidante & Preparado al $75 \%$ \\
\hline Tegosivin HL 100 & Goldschmidt & Organosilícico polímero & Hidrófugo & Al $5 \%$ en Xileno \\
\hline Consolidante $\mathrm{OH}$ & Wacker & Silicato de etilo & Consolidante & Preparado al $75 \%$ \\
\hline ARD 55.050 & Raccanello & Polímero acrilsilicónico & Consolidante + Hidrófugo & Preparado al $10 \%$ \\
\hline
\end{tabular}

I. Compatibilidad de los productos y técnicas empleados con los materiales originales de la obra o con otros productos o técnicas de tratamiento que se vayan a utilizar.

2. Eficacia del tratamiento, es decir, que con él se consiga el fin que se persigue.

3. Resistencia a los agentes de alteración que actuarán sobre la obra una vez restaurada.

\section{Tratamientos estudiados}

Dado el estado de decohesión en que se encontraba la piedra era necesario aplicar un tratamiento de consolidación; por otra parte, la exposición a la lluvia del monumento hacía también necesario protegerlo de la entrada del agua líquida, aplicando un hidrófugo.

Teniendo en cuenta el gran número de productos existentes en el mercado, se consideró necesario estudiar algunos previamente a su aplicación, para elegir aquéllos que mayores garantías de éxito ofreciesen. Se incluyeron en el estudio aquellos tratamientos que, tanto por experiencia propia como por referencias bibliográficas, proporcionan generalmente buenos resultados.

Para la realización de los distintos ensayos se han utilizado probetas cúbicas de $5 \mathrm{~cm}$, obtenidas de sillares desprendidos del muro lateral a causa de las lluvias. Antes de la impregnación con los productos, las probetas se han limpiado y dejado secar al aire, para conseguir un contenido de humedad higroscópica en equilibrio con el ambiente. Las probetas se han tratado por inmersión durante diez minutos, tiempo suficiente para conseguir una buena penetración. Tras la impregnación se ha controlado la evolución de peso de las probetas hasta el completo secado de los tratamientos, para determinar el tiempo de secado y el incremento de peso, datos que pueden ser útiles de cara a la aplicación in situ.

Los dos consolidantes tardan I5-20 días en secarse por completo, ya que estos productos se aplican en forma de monómeros y deben polimerizar en la piedra. El hidrófugo y el acrilsilicónico, que ya están polimerizados, tardan algo menos en completar el proceso, unos 6-7 días.

\section{Estudio experimental}

\section{Compatibilidad con el material}

Para determinar si un tratamiento es aceptable, es fundamental conocer cómo modifica algunas características del material. Entre ellas son muy importantes la porosidad y la velocidad de evaporación de agua. Si la variación producida es excesivamente alta, puede ser necesario descartar dicho tratamiento, ya que en este caso la zona tratada (que siempre es una capa superficial más o menos fina) tendría características muy diferentes del resto del material no tratado y en la interfase entre ambos se producirín fenómenos que podrían acabar con el desprendimiento de la capa tratada.

\begin{tabular}{lcc}
\hline \multicolumn{3}{l}{ Tabla 4. Porosidad media (\%) con y sin tratamiento } \\
\hline Tratamiento & Porosidad antes / después & Disminución de porosidad (\%) \\
\hline Tegovakon V & $29,93 / 27,75$ & 7,28 \\
\hline Tegosivin HL 100 & $32,22 / 27,74$ & 13,90 \\
\hline Consolidante OH & 31,83 / 29,04 & 8,76 \\
\hline ARD 55050 & $33,34 / 30,39$ & 8,84 \\
\hline
\end{tabular}

\section{Fig. 10 Desorción de agua}

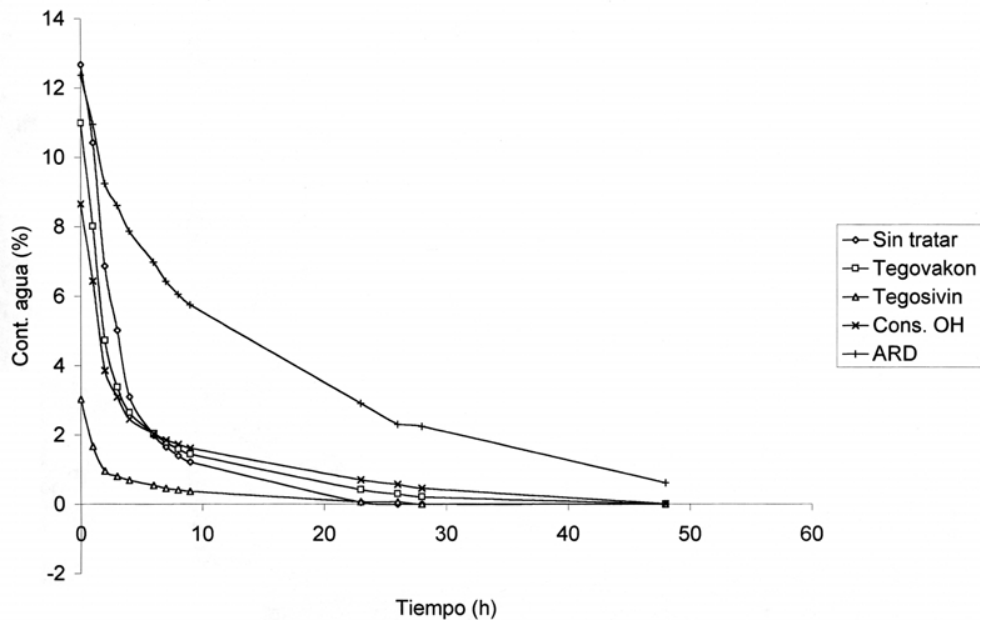




\section{Porosidad}

La determinación de la porosidad abierta se ha realizado por el método de absorción de agua a vacío (Van Keulen 1972). La porosidad se ha determinado en las probetas antes y después del tratamiento y los valores medios aparecen en la Tabla 4, así como la disminución media de la porosidad.

\section{Desorción de agua}

El estudio del secado del material se ha realizado según el ensayo propuesto dentro del Programa de Normalización de Estudios previos del IAPH (Ontiveros 1998). Las probetas se saturan de agua por

\section{Fig. 11 Absorción de agua por capilaridad}

\section{Figura 11. Absorción de agua por capilaridad}

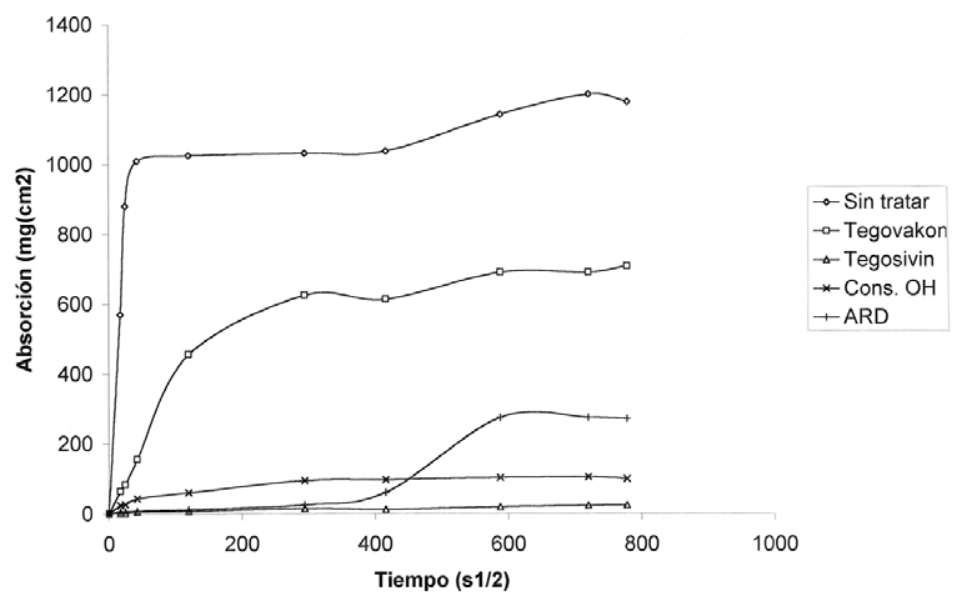

Fig. 12 Absorción por inmersión

Figura 12. Absorción por inmersión

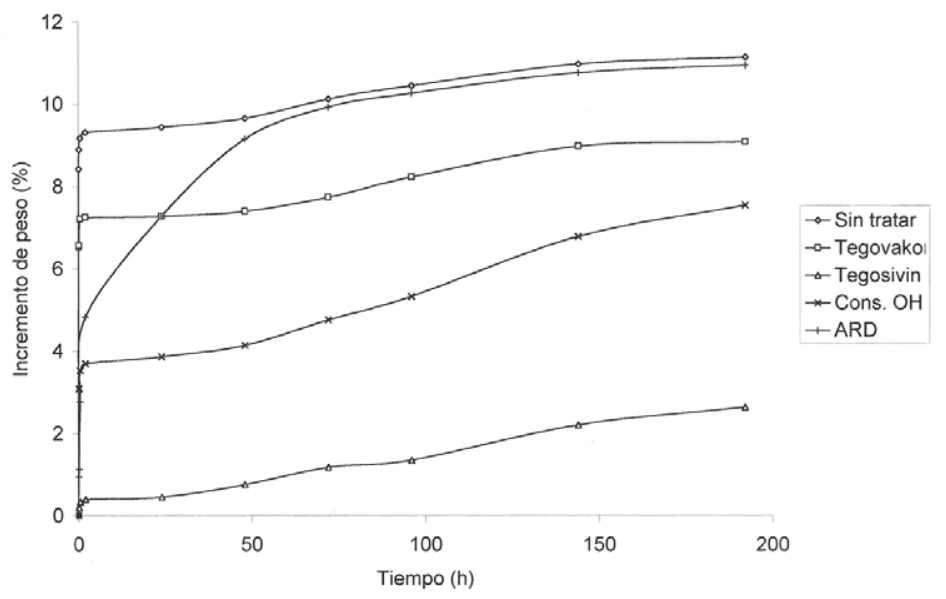

Tabla 5 Incremento de dureza

Tratamiento Consolidante $\mathrm{OH}$ Tegovakon Tegosivin HL100 ARD 55.050

\begin{tabular}{lllll}
\hline Incr. dureza (\%) & 18,7 & 16,5 & 13,7 & 14,9 \\
\hline
\end{tabular}

inmersión total durante siete días. Después de determinar el peso saturado se mantienen en cámara climática a $20 \pm 2^{\circ} \mathrm{C}$ y $50 \pm 5 \%$ de humedad relativa, pesándose periódicamente. En la figura 10 se representan los valores medios del contenido de agua (expresado como \% respecto del peso seco) para las probetas de cada tipo en función del tiempo.

En el proceso de secado pueden observarse dos fases. Para contenidos de agua elevados (primera parte del ensayo), la evaporación se produce en la superficie del material con una velocidad constante; cuando el contenido de agua desciende por debajo de cierto valor, denominado contenido crítico, la evaporación se produce en el interior de la piedra y el agua sale al exterior en forma de vapor, por lo que el proceso se ralentiza. Normalmente, tanto la velocidad de evaporación como el contenido crítico se ven afectados por la aplicación de tratamientos, siempre de forma negativa, es decir, la velocidad de secado disminuye y el contenido crítico aumenta, con lo que la piedra permanece con mayor contenido de agua durante más tiempo.

En la figura 10 puede observarse que los tratamientos organosilícicos se comportan prácticamente igual que la piedra sin tratar, mientras que en el caso del acrilsilicónico se produce una acusada disminución de la velocidad de secado y un incremento del contenido crítico.

\section{Eficacia del tratamiento}

Cuando se aplica un tratamiento se pretende conseguir una mejora en determinadas características. Con los productos hidrófugos se trata de disminuir la entrada de agua en la piedra y con los consolidantes aumentar la cohesión del material. Para evaluar esta mejora se determina, en el primer caso, cómo se modifica la absorción de agua y, en el segundo, se miden las propiedades mecánicas.

\section{Absorción de agua por capilaridad}

La determinación de la absorción de agua por capilaridad se ha realizado siguiendo la RECOMANDAZIONE NORMAL I |/85. En la Figura I | se representa la absorción de agua (valores medios de incremento de peso, $\mathrm{mg} / \mathrm{cm}^{2}$ ) frente a la raíz cuadrada del tiempo $\left(\mathrm{s}^{1} / 2\right)$.

Se observa cómo las probetas tratadas con Tegovakon absorben algo menos que las no tratadas, lo cual se debe fundamentalmente a la disminución de porosidad de estas probetas. El otro consolidante organosilícico produce una disminución mucho más acusada, lo cual puede deberse a restos orgánicos sin polimerizar o a pequeñas cantidades de disolvente que no ha terminado de evaporarse.

El hidófugo presenta un buen comportamiento hidrorrepelente, que se mantiene sin alteración a lo largo del ensayo. Por último, ARD (consolidante e hidrófugo) se comporta bien inicialmente, pero a medio plazo comienza a aumentar la cantidad de agua absorbi- 
da; es decir, pierde parcialmente sus cualidades hidrófugas tras un contacto prolongado con agua.

\section{Absorción de agua por inmersión}

La determinación de la absorción de agua por capilaridad se ha realizado siguiendo la RECOMANDAZIONE NORMAL 7/8I. En la Figura 12 se representan los valores medios de absorción (incremento de peso, \%) para cada tratamiento y para las muestras sin tratar.

Las probetas tratadas con ARD absorben prácticamente la misma cantidad que las no tratadas, aunque la velocidad de absorción inicial es algo menor, es decir, este tratamiento prácticamente no ejerce efecto hidrófugo cuando el aporte de agua es grande. Tegovakon disminuye ligeramente la absorción, mientras que Consolidante $\mathrm{OH}$ la reduce en mayor porcentaje aunque sin llegar a estabilizarse.

\section{Propiedades mecánicas}

El efecto de los consolidantes se pone de manifiesto en una mejora de las características mecánicas, pudiéndose estudiar de dos formas: medida de la dureza superficial o medida de la resistencia a compresión. En este caso se ha preferido esta última, ya que el efecto de los tratamientos normalmente se manifiesta en una capa superficial.

La medida de la dureza superficial que se ha realizado se basa en la resistencia que opone un material a ser penetrado por un cuerpo más duro. La dureza de las probetas tratadas y sin tratar se ha medido con un durómetro tipo Rockwell, empleando una bola de $5 \mathrm{~mm}$, aplicando una precarga de $10 \mathrm{Kg}$ y una carga total de $30 \mathrm{Kg}$. El valor de la dureza se determina como función inversa de la diferencia entre la penetración de la bola con la carga de $30 \mathrm{Kg}$ y con la carga de $10 \mathrm{Kg}$. En cada probeta se han realizado 9 medidas en cada una de las tres caras que confluyen en un vértice, en total 27 medidas.

\section{Resistencia a la alteración. Ensayos de alteración acelerada}

El último paso en la evaluación de los tratamientos consiste en someter las probetas a condiciones que simulen los mecanismos de alteración observados en el edificio, pero de forma concentrada en el tiempo, para obtener resultados en plazos más breves. En este caso, los mecanismos observados son dos:

- alternancia entre la cristalización y solubilización de las sales presentes en la piedra, que pueden proceder de varias fuentes: los morteros, la propia piedra, o el subsuelo.

- oscilaciones en el contenido de humedad del material, al variar las condiciones de temperatura y humedad ambientales.

Por ello, los ensayos de alteración acelerada que mejor reproducen dichos mecanismos son el de cristalización de sales y el de humedad-sequedad.

\section{Ensayo de cristalización de sales}

Este ensayo consiste en someter a las probetas a cristalizaciones y solubilizaciones sucesivas de una sal soluble. Se ha realizado siguiendo el ensayo propuesto dentro del Programa de Normalización de Estudios previos del IAPH (Villegas 2000). Se ha utilizado una solución de sulfato sódico al $10 \%(\mathrm{p} / \mathrm{v})$ y se han realizado ciclos formados por las siguientes fases:

- Fase de inmersión: 24 horas de inmersión en la solución

- Fase de secado: $\quad 22$ horas de secado a $65^{\circ} \mathrm{C}$ en estufa

2 horas de enfriamiento y pesada

Se emplea esta sal porque puede cristalizar con 10 moléculas de agua dentro de su estructura cristalina, con lo que se produce un aumento de volumen de alrededor del $300 \%$; de esta forma se provocan tensiones en el interior de los poros de la piedra que causan la disgregación de la misma.

\section{Fig. 13 Ensayo de cristalización de sales}

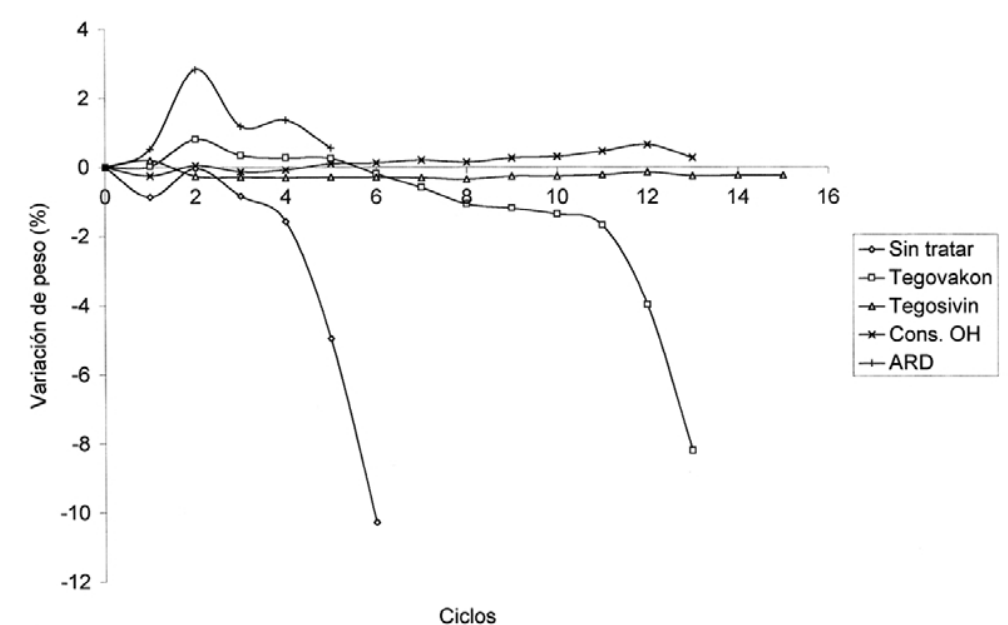

Ciclos

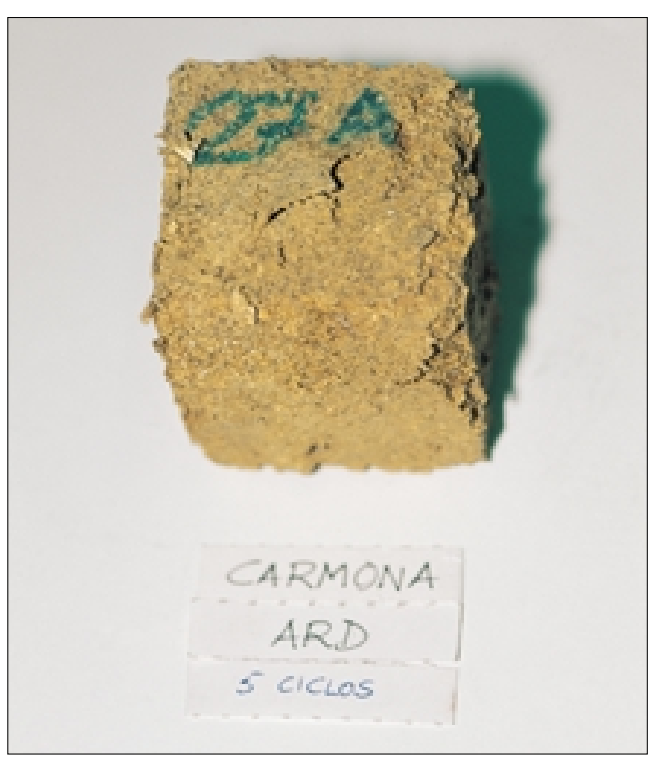

Fig. 14 Probeta tratada con ARD tras 5 ciclos de cristalización de sales 
A lo largo del ensayo se efectúa el control del peso de las probetas en cada ciclo y de las alteraciones macroscópicas que van apareciendo. Cuando el grado de deterioro alcanzado por una probeta es grande se elimina del ensayo. En la figura 13 se representa la variación media de peso (en \% respecto al peso inicial) para cada grupo de probetas.

Tegosivin HL 100 impide la entrada de solución en las probetas, por lo que no llegan a producirse alteraciones. Tegovakon y Consolidante $\mathrm{OH}$ reducen la velocidad de alteración, especialmente este último. ARD se comporta inicialmente bien, pero tras un corto número de ciclos las probetas sufren el desprendimiento de una fina capa superficial. Este hecho se produce cuando los tratamientos se acumulan en la zona más externa de la piedra y dificultan el paso de agua, acumulándose las sales en la interfase. En la figura 14 se muestra una de estas probetas tras cinco ciclos.

\section{Ensayo de humedad-sequedad}

Este ensayo consiste en someter a las probetas a ciclos de humidificación y secado. Se ha realizado siguiendo la propuesta del Programa de Normalización de Estudios previos del IAPH (Villegas 2000). Los ciclos están formados por las siguientes fases:

- Fase de humidificación: 24 horas de inmersión en agua a temperatura ambiente

- Fase de secado:

22 horas de secado a $80^{\circ} \mathrm{C}$ en estufa 2 horas de enfriamiento y pesada

Se han realizado 20 ciclos, en los cuales no han aparecido alteraciones en ninguna de las probetas, igual que se ha observado en otros ensayos semejantes (Villegas 2000b).

\section{Conclusiones}

La piedra utilizada en la construcción de la Puerta de Córdoba corresponde a las calcarenitas amarillas de la Formación del Alcor. La piedra es muy similar a la de las canteras estudiadas, apareciendo en el edificio sillares de textura y estructura con las mismas heterogeneidades que aparecen en cantera, lo que hace dificil establecer su origen exacto.

El alto grado de alteración observado en algunas zonas de la Puerta (principalmente en las zonas bajas), puede ser atribuido a cambios cíclicos en el contenido en humedad junto a la presencia de arcillas expansivas y yeso.

En la elaboración de hormigones, morteros y enlucidos parece haberse utilizado preferentemente materiales locales con diferencias en los contenidos de cal añadidos. Los únicos materiales que presentan características bien diferenciadas son los revocos y enlucidos de época romana en los que se empleó marmolina como árido, al igual que los morteros de unión del Siglo XVIII han sido los únicos en los que se empleó yeso (y cal).

La metodología aplicada en la evaluación de tratamientos ha permitido establecer las diferencias entre los tratamientos y seleccionar el que proporciona mejores resultados. Entre los consolidantes, el comportamiento de los organosilícicos es semejante y mucho mejor que el de ARD, destacando ligeramente Consolidante $\mathrm{OH}$. De los dos hidrófugos, sobresale significativamente Tegosivin HL 100.

\section{Bibliografía}

ADAMS A.E., MACKENZIE W.S. \& GUILFORD. C (1994): Atlas of Sedimentary Rocks under Microscope. Longman Scienfic \& Technical

ASHURST, J. and ASHURST, N. (1988): Mortars, Plasters and Renders. Practical Building Conservation Series. Gower Technical Press Ltd. England

BERZOSA, J. and MARTIN, A. "Análisis rápido de materiales calizos y silíceos. Ensayos e Investigación (1966).

ESBERT, R.M.; ORDAZ, J.: Glosario de términos relacionados con el deterioro de piedras de construcción. Materiales de construcción Vol. 28 n² 209 (39-45), 1986

FOLK, R.L.(1974): Petrology of Sedimentary Rocks. Hemphills, Austin, Texas.

I.G.M.E (1988). Mapa geológico de España. Hoja 985. Carmona.

RODRÍGUEZ NAVARRO C (1994): Causas y mecanismos de alteración de los materiales calcáreos de las catedrales de Granada y Jaén. Tesis Doctorales Universidad de Granada [inédita]

VILLEGAS SÁNCHEZ, R. (1996): Ensayos y técnicas para evaluar la eficacia de los tratamientos. En Técnicas de diagnóstico aplicadas a la conservación de los materiales de construcción en los edificios históricos. Sevilla: Instituto Andaluz del Patrimonio Histórico, 1996 (Cuadernos Técnicos ; 2), pp. 151-160.
VAN KEULEN, H.: Determination of maximun water content. Report n. BI-72-39. June 1972

ONTIVEROS ORTEGA, E.; VILLEGAS SÁNCHEZ, R. (1998): Programa de normalización de estudios previos y control de calidad en las intervenciones. Propiedades hídricas, $2^{\text {a }}$ parte. $\mathrm{PH}$. Boletín del IAPH, $n^{\circ} 23$, junio 1998, pp. 40-45.

NORMAL I I/85. Assorbimento d'acqua per capillaritá. Coefficiente di assorbimento capillare. C.N.R. - I.C.R. Roma. 1985.

NORMAL 7/81. Assorbimento d'acqua per immersione totale. Capacitá di imbibizione. C.N.R. - I.C.R. Roma. 1981.

VILLEGAS SÁNCHEZ, R. (2000): Programa de normalización de estudios previos y control de calidad en las intervenciones. Ensayos de alteración de materiales pétreos. $\mathrm{PH}$. Boletín del IAPH, $\mathrm{n}^{\circ}$ 31, junio 2000, pp. 78-88.

VILLEGAS SÁNCHEZ, R.; ESPINOSA GAITÁN, J.; ALCALDE MORENO, M. (2000). Study of weathering factors and evaluation of treatments for the stones of Santa María de la Encarnación Church, Constantina (Sevilla, Spain). Proc. 9th International Congress. on Deterioration and Conservation of Stone. 697-705, Venice, june, 2000. 